\title{
On tuning a reactive silencer by varying the position of an internal membrane ${ }^{\text {a) }}$
}

\author{
Jane B. Lawrie ${ }^{\text {b) }}$ and Idil M. M. Guled \\ School of Information Systems, Computing and Mathematics, Mathematical Sciences, Brunel University, \\ Uxbridge UB8 3PH, United Kingdom
}

(Received 7 August 2005; revised 4 April 2006; accepted 19 May 2006)

\begin{abstract}
A mode-matching method is used to investigate the performance of a two-dimensional, modified reactive silencer. The modification takes the form of a membrane which is attached to the internal walls of the expansion chamber parallel to the axis of the inlet/outlet ducts. The height of the membrane above the level of the inlet/outlet ducts can be varied and, by this means, the device is tuned. It is shown that the stopband produced by the silencer can be broadened and/or shifted depending upon the height to which the membrane is raised. Attention is focused on the efficiency of the device at low-frequencies-the regime where dissipative silencers are usually least effective. The potential use of the device as a component in a hybrid silencer for heating ventilation and air-conditioning (HVAC) ducting systems is discussed. (C) 2006 Acoustical Society of America.
\end{abstract}

[DOI: $10.1121 / 1.2213571]$

PACS number(s): 43.50.Gf, 43.20.Mv, 43.40.Dx [DKW] Pages: 780-790

\section{INTRODUCTION}

Ducted fan noise is an issue that affects a diverse range of industrial applications: the generation of "buzz-saw" noise in aero-engines; ${ }^{1}$ noise emission from chimney stacks of power stations; ${ }^{2}$ and heating ventilation and air-conditioning (HVAC) systems. ${ }^{3}$ Dissipative devices such as acoustic linings or silencers are often used to reduce the noise levels. These work well in the mid- and high-frequency range but are less effective for low-frequency noise. In many applications, therefore, it is the low-frequency tonal fan noise that is the most pervasive.

Tonal fan noise tends to be in the range $100-500 \mathrm{~Hz}$ and restricted to a narrow band which, depending on the application, can be as large as $50 \mathrm{~Hz}$ (Ref. 2) or as little as $6 \mathrm{~Hz} .{ }^{4}$ In recent years much research has been carried out on ways of reducing such noise. Passive devices that perform at low frequencies include expansion chambers and Helmholtz resonators. Both, however, are of limited value in practical situations, such as HVAC installations, where constraints on space rule out the use of bulky devices. Further, in the case of the Helmholtz resonator, the transmission loss is modest, typically in the region of $6 \mathrm{~dB},{ }^{5}$ and the frequency range over which it is effective is narrow. The latter point has been addressed and various designs for an adaptive Helmholtz resonator have been proposed; see for example de Bedout $e t$ $a l .{ }^{6}$ While Helmholtz resonators, both adaptive and standard, have found application in a range of industries, ${ }^{5,7}$ they are not, to the authors's knowledge, extensively used in HVAC systems.

\footnotetext{
${ }^{a}$ Portions of this work were presented in Mohamed-Guled and Lawrie, "A parametric investigation of the acoustic power in a two-dimensional waveguide with membrane bounded cavity," in Proceedings of IUTAM 2002/4, edited by A. B. Movchan (Kluwer, Dordrecht, 2004). Liverpool, U.K July 2002.

b) Author to whom correspondence should be addressed. Electronic mail: jane.lawrie@brunel.ac.uk
}

An alternative to passive noise control is offered by active devices which aim at cancellation of the noise. This is a fast-growing area of research and it is clear that impressive levels of noise reduction are possible for a wide range of applications. ${ }^{3,2,4}$ Such devices are, however, not without potential problems. They all require additional energy (and thus additional expense) to achieve noise reduction and, further, the loudspeakers may occupy space in the region exterior to the duct. For example, the housing for the active silencer cassette in the configuration considered by $\mathrm{Krüger}^{3}$ is spacewise equivalent to a rather bulky expansion chamber. In situations where space is limited, this extra height would be undesirable. Furthermore, in all such devices there is the possibility of creating more noise should the control algorithm fail.

In this paper a generalization of the reactive silencer recently investigated by Huang ${ }^{8-10}$ is considered with a view to assessing its potential as a means of reducing tonal fan noise in HVAC systems. The device comprises a twodimensional reactive silencer in which a membrane is attached to the internal walls of the expansion chamber parallel to the axis of the inlet/outlet ducts. The height of the membrane above the level of the inlet/outlet ducts can be varied, and the device is tuned by selecting the membrane height that gives the widest stopband for a specified frequency. The concept of utilizing flexible panels as a means of controlling low-frequency noise is by no means new. As long ago as 1963 Dowell and Voss ${ }^{11}$ studied the vibrations of a cavitybacked panel in the presence of flow. More recently, and in the context of architectural acoustics, Kang and Fuchs ${ }^{12}$ have investigated the effectiveness of cavity-backed microperforated membranes as an acoustic absorber.

The model problem is solved using a mode-matching technique. The fluid velocity potentials in the inlet and outlet ducts are expressed in the form of standard Fourier cosine series. In contrast, due to the presence of high-order spacial 
derivatives in the membrane boundary condition, the eigensystem of the silencer region is non-Sturm-Liouville. Further, the eigenfunctions are piecewise continuous. Nevertheless, the system satisfies a known orthogonality relation. ${ }^{13,14}$ This, together with the usual orthogonality property for $\{\cos (n \pi y / a) \mid n=0,1,2, \ldots\}$, enables continuity of pressure/ normal velocity to be imposed at the mouths of the inlet/ outlet ducts, and appropriate edge conditions to be applied where the membrane joins the structure. The two resulting systems of algebraic equations, formulated in terms of the amplitudes of the reflected and transmitted waves, are truncated and solved numerically. In this article the roots of the characteristic function for the modified expansion chamber are determined numerically before the mode-matching equations are truncated and inverted. For a nondissipative system, such as this, root finding usually presents few problems. It is worthwhile mentioning, however, that the boundary value problem for the model problem falls within the class whereby the mode-matching equations can be recast into root-free form, ${ }^{15}$ which bypasses the root-finding process.

In contrast, the method employed by Huang ${ }^{8,9}$ automatically avoids the need for root finding. He employs a technique whereby the sound fields within the duct and cavity are represented by an infinite sum of Fourier integrals, each one forced by a velocity distribution $\sin [n \pi(x+\ell) /(2 \ell)]$ on the duct surface for $-\ell \leqslant x \leqslant \ell$. The Fourier coefficients of the pressure field are then determined by substituting an evaluated form of the Fourier integrals into the membrane condition. This works well for the case in which the membrane lies at the mouth of the expansion chamber in line with the upper boundary of the inlet/outlet ducts. The method cannot, however, be extended to the more general situation in which the membrane lies above or below this level.

The aims of this article are to investigate the effects of varying the height of the membrane within the expansion chamber; to discuss the the potential for using this device as a component of a hybrid silencer for HVAC ducting systems; to compare Huang's solution method with the modematching method, and to discuss the relative merits of the two approaches. In Sec. II the traveling wave forms for the expansion chamber region are discussed and the appropriate orthogonality relation is stated. This is used in Sec. III, where the boundary value problem corresponding to acoustic transmission through the modified reactive silencer is stated and solved using the mode-matching technique outlined above. Section IV is concerned with the derivation of a lowfrequency approximation to the solution. This proves to be surprisingly accurate and is a useful tool in terms of verifying the results obtained via mode matching. In Sec. V numerical results are presented. Of particular interest are those showing transmission loss against frequency for silencers with low aspect ratio (i.e., those in which the ratio of silencer length to expansion chamber height is less than three). It is found that such silencers exhibit only modest stopbands when the membrane is positioned at the mouth of the expansion chamber; however, by altering the position of the membrane they can be tuned and the width of the stopband can be increased by up to $38 \%$. Finally, Sec. VI presents a discussion of the results and potential extensions to the theory.

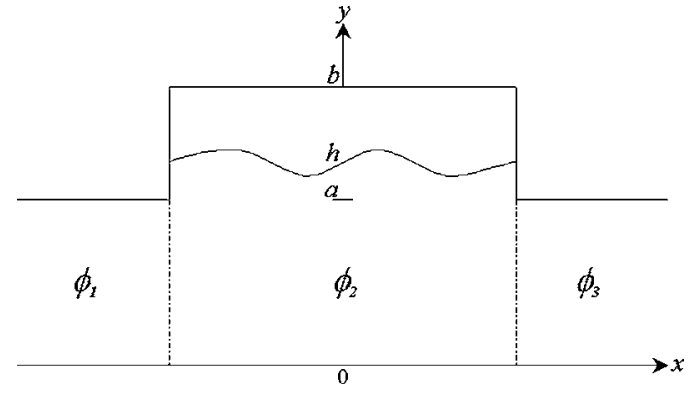

FIG. 1. Silencer geometry.

\section{TRAVELING WAVE SOLUTIONS}

In this section a detailed discussion of the traveling wave forms of the silencer region (see Fig. 1) is presented. It is appropriate, therefore, to consider the unforced boundary value problem governing the fluid velocity potential within an infinite rigid duct, of height $\bar{b}$, in which a horizontal membrane is situated along $\bar{y}=\bar{h},-\infty<\bar{x}<\infty$, with $0<\bar{h}<\bar{b}$. Harmonic time dependence, $e^{-i \omega \bar{t}}$, where $\omega$ is the radian frequency, is assumed. Thus, the time-dependent fluid velocity potential, $\bar{\Phi}$, can be expressed as $\bar{\Phi}(\bar{x}, \bar{y}, \bar{t})=\bar{\phi}(\bar{x}, \bar{y}) e^{-i \omega \bar{t}}$ and, henceforth, the time-independent potential $\bar{\phi}$ will be used. It is convenient to nondimensionalize the boundary value problem using typical length and time scales $k^{-1}$ and $\omega^{-1}$, where $k=\omega / c$. Thus, the nondimensional quantities $x, y$, etc. will henceforth be used. These are related to their dimensional counterparts by $x=k \bar{x}, y=k \bar{y}$, etc.

The velocity potential satisfies Helmholtz's equation with unit wave number, that is

$$
\left(\nabla^{2}+1\right) \phi=0 .
$$

At the rigid walls the normal component of velocity potential is zero,

$$
\frac{\partial \phi}{\partial y}=0, \quad y=0, b, \quad-\infty<x<\infty .
$$

The membrane at $y=h,-\infty<x<\infty$, is described by the condition

$$
\left(\frac{\partial^{2}}{\partial x^{2}}+\mu^{2}\right) \frac{\partial \phi}{\partial y}-\alpha[\phi(x, y)]_{h^{-}}^{h^{+}}=0, \quad y=h,
$$

where $\mu$ and $\alpha$ are the nondimensional in vacuo membrane wave number and the nondimensional fluid loading parameter, respectively. These quantities are defined by $\mu=c / c_{m}$ and $\alpha=c^{2} \rho /(k T)$, where $c_{m}=\left(T / \rho_{m}\right)^{1 / 2}$ is the in vacuo speed of waves on the membrane, $T$ is the membrane tension, and $\rho_{m}$ is the mass per unit area of the membrane.

On using the usual process of separation of variables, it is found that the eigenmodes for the silencer region are

$$
\phi_{n}=Y_{n}(y) e^{ \pm i \nu_{n} x}, \quad 0 \leqslant y \leqslant b,
$$

where

$$
Y_{n}(y)=\left\{\begin{array}{l}
\cosh \left(\gamma_{n} y\right), \quad 0 \leqslant y \leqslant h \\
\xi_{n} \cosh \left[\gamma_{n}(b-y)\right], \quad h<y \leqslant b
\end{array},\right.
$$

and 


$$
\xi_{n}= \begin{cases}-\frac{\sinh \left(\gamma_{n} h\right)}{\sinh \left[\gamma_{n}(b-h)\right]}, & n \neq 1 \text { and } \gamma_{n} \neq \frac{i j \pi q}{p h} \\ 1, & n=1 \\ (-1)^{j}, & n \neq 1 \text { and } \gamma_{n}=\frac{i j \pi q}{p h}\end{cases}
$$

with $\gamma_{n}^{2}-\nu_{n}^{2}+1=0$. This expression encompasses both fluidmembrane coupled waves and uncoupled modes. For the coupled modes, the eigenvalues, $\gamma_{n}^{c}, n=0,1,2, \ldots$, are the roots of the characteristic equation

$$
K(\gamma)=\left(\gamma^{2}+1-\mu^{2}\right) \gamma \sinh (\gamma h)-\alpha \frac{\sinh (\gamma b)}{\sinh [\gamma(b-h)]}=0 .
$$

Note that $K(\gamma)$ is an even function of $\gamma$. It is convenient, therefore, to denote the roots of (7) by $\pm \gamma_{n}^{c}, n=0,1,2, \ldots$ and discuss only the positive roots (i.e., those lying in the upper half of the complex gamma plane axes, which is deemed to include the positive real axis). It is found that there is always one real root, $\gamma_{0}^{c}>0$, and an infinite number of imaginary roots, $\gamma_{n}^{c}, n=1,2,3, \ldots$, which are labeled with increasing distance up the imaginary axis. In addition to the fluid membrane modes there are other possible waveforms for the silencer region. First is the plane acoustic wave $e^{i x}$. This is a trivial solution to (3) and it follows that this mode can always exist without interaction with the membrane. Other uncoupled modes exist only if the silencer height $b$ can be expressed in the form $b=p h / q$, for integer values of $p$ and $q$. The modes of interest are a subset of the usual rigid duct modes: those that have zero velocity normal to the membrane at $y=h$. Thus, admissible wave numbers for the uncoupled modes are defined by $\gamma_{1}^{\mu}=0$ (always present) and $\gamma_{j}^{\mu}=i j \pi q /(p h), j=p, 2 p, 3 p, \ldots$ (present only for $b=p h / q$ ). Note that these wave numbers are not roots to the characteristic equation (7).

The set of all admissible wave numbers is the union of those for the coupled and uncoupled modes, that is, $\left\{\gamma_{n} \mid n=0,1,2 \ldots\right\}=\left\{\gamma_{m}^{c} \mid m=0,1,2, \ldots\right\} \cup\left\{\gamma_{j}^{\mu} \mid j=1, p, 2 p, \ldots\right\}$. They are ordered $\gamma_{0}=\gamma_{0}^{c}, \gamma_{1}=\gamma_{1}^{u}$ and then by increasing imaginary part. The functions $Y_{n}(y)$ defined by (5) and (6) satisfy the following orthogonality relation: ${ }^{14}$

$$
Y_{n}^{\prime}(h) Y_{m}^{\prime}(h)+\alpha \int_{0}^{b} Y_{n}(y) Y_{m}(y) d y=E_{n} \delta_{m n},
$$

where the prime indicates differentiation with respect to $y$ and $\delta_{m n}$ is the usual Kronecker delta. For fluid-coupled modes the quantity $E_{n}$ is given by

$$
E_{n}=\left.\frac{Y_{n}^{\prime}(h)}{2 \gamma_{n}} \frac{d}{d \gamma} K(\gamma)\right|_{\gamma=\gamma_{n}},
$$

whereas for the uncoupled modes

$$
E_{n}=\frac{\alpha b}{2} \varepsilon_{n-1},
$$

where $\varepsilon_{n}=2$ for $n=0$ and 1 otherwise. Equations (9) and (10) can be rearranged for the general case as

$$
E_{n}=\frac{\alpha}{2}\left[e_{n}(h)+\xi_{n}^{2} e_{n}(b-h)\right],
$$

where

$$
e_{n}(x)=x+\frac{\left(3 \gamma_{n}^{2}+1-\mu^{2}\right)}{2 \gamma_{n}\left(\gamma_{n}^{2}+1-\mu^{2}\right)} \sinh \left(2 \gamma_{n} x\right) .
$$

\section{THE BOUNDARY VALUE PROBLEM}

The mode-matching solution for acoustic transmission through the modified silencer is presented in this section. The model comprises rigid inlet and outlet ducts occupying the regions $0 \leqslant y \leqslant a,|x|>\ell$ of a Cartesian frame of reference, together with a finite duct of height $b>a$ in the gap $|x| \leqslant \ell$. The structure is closed by two vertical rigid surfaces at $x$ $= \pm \ell, a \leqslant y \leqslant b$, forming a rectangular expansion chamber. The interior region of the duct contains a compressible fluid of sound speed $c$ and density $\rho$. The fluid in the expansion chamber is separated into two regions by a horizontal membrane which lies along $y=h, h \geqslant a,|x| \leqslant \ell$; see Fig. 1 .

A multimodal incident field, with harmonic time dependence, is incident in the positive $x$ direction towards $x=-\ell$ where it is scattered. It is convenient to split the velocity potential into three parts corresponding to the inlet duct, the expansion chamber, and the outlet duct, respectively. The time-independent fluid velocity potentials for each duct region are thus $\phi_{1}(x, y), x<0 ; \phi_{2}(x, y), 0<x<2 \ell$, and $\phi_{3}(x, y), x>2 \ell$. The velocity potentials, $\phi_{j}, j=1,3$, satisfy the nondimensionalized Helmholtz's equation (1) with boundary conditions

$$
\begin{aligned}
& \frac{\partial \phi_{1}}{\partial y}=0, \quad y=0, a, \quad-\infty<x \leqslant-\ell, \\
& \frac{\partial \phi_{3}}{\partial y}=0, \quad y=0, a, \quad \ell \leqslant x<\infty,
\end{aligned}
$$

whereas the potential $\phi_{2}$ satisfies Eqs. (1)-(3) together with

$$
\frac{\partial \phi_{2}}{\partial x}=0, \quad x= \pm \ell, \quad a<y \leqslant b,
$$

and the edge condition

$$
\phi_{2 y}( \pm \ell, h)=0,
$$

where the subscript $y$ indicates differentiation with respect to that variable. The eigenfunction expansions for $\phi_{j}, j$ $=1,2,3$ are

$$
\phi_{1}=\sum_{n=0}^{\infty} F_{n} \cos \left(\frac{n \pi y}{a}\right) e^{i \eta_{n}(x+\ell)}+\sum_{n=0}^{\infty} A_{n} \cos \left(\frac{n \pi y}{a}\right) e^{-i \eta_{n}(x+\ell)},
$$

$$
\phi_{2}=\sum_{n=0}^{\infty}\left(B_{n} e^{i \nu_{n} x}+C_{n} e^{-i \nu_{n} x}\right) Y_{n}(y)
$$


$\phi_{3}=\sum_{n=0}^{\infty} D_{n} \cos \left(\frac{n \pi y}{a}\right) e^{i \eta_{n}(x-\ell)}$,

where $Y_{n}(y)$ is given by (5). Here, $F_{n}$ are the amplitudes of the incident modes while $A_{n}, D_{n}$ are the complex amplitudes of the $n$th reflected, transmitted modes, respectively and $B_{n}, C_{n}$ are the amplitudes of the modes in the silencer region. Note that $\eta_{n}=\left(1-n^{2} \pi^{2} / a^{2}\right)^{1 / 2}$ and $\nu_{n}=\left(\gamma_{n}^{2}+1\right)^{1 / 2}, n$ $=0,1,2, \ldots$. The focus of this article is on transmission loss at low frequencies and, as such, plane-wave forcing is of primary interest. To broaden the applicability of the article, however, the model problem has been formulated for a multimodal incident field. For plane-wave forcing $F_{n}=\delta_{n 0}, n=0,1,2, \ldots$, whereas the appropriate form of the modal amplitudes in the case of, for example, equal modal energy density, are given by Mechel. ${ }^{16}$

The coefficients $A_{n}, B_{n}, C_{n}$, and $D_{n}$ are determined by matching the fluid pressure and the normal velocity at the interfaces $x= \pm \ell, 0 \leqslant y \leqslant a$. Continuity of pressure is expressed as

$$
\phi_{1}(-\ell, y)=\phi_{2}(-\ell, y), \quad 0 \leqslant y \leqslant a,
$$

and

$$
\phi_{2}(\ell, y)=\phi_{3}(\ell, y), \quad 0 \leqslant y \leqslant a \text {. }
$$

Similarly, continuity of normal velocity gives

$$
\frac{\partial \phi_{2}}{\partial x}(-\ell, y)=\left\{\begin{array}{l}
\frac{\partial \phi_{1}}{\partial x}(-\ell, y), \quad 0 \leqslant y \leqslant a \\
0, \quad a<y \leqslant b
\end{array},\right.
$$

and

$$
\frac{\partial \phi_{2}}{\partial x}(\ell, y)=\left\{\begin{array}{l}
\frac{\partial \phi_{3}}{\partial x}(\ell, y), \quad 0 \leqslant y \leqslant a \\
0, \quad a<y \leqslant b
\end{array} .\right.
$$

On substituting (17)-(19) into (20) and (21) and making use of the orthogonality relation for $\{\cos (n \pi y / a)\}$, it is found that

$$
A_{n}+F_{n}=\frac{2}{a \epsilon_{n}} \sum_{m=0}^{\infty}\left(B_{m} e^{-i \nu_{m} \ell}+C_{m} e^{i \nu_{m} \ell}\right) R_{n m}
$$

and

$$
D_{n}=\frac{2}{a \epsilon_{n}} \sum_{m=0}^{\infty}\left(B_{m} e^{i \nu_{m} \ell}+C_{m} e^{-i \nu_{m} \ell}\right) R_{n m},
$$

where

$$
R_{n m}=\int_{0}^{a} \cos \left(\frac{n \pi y}{a}\right) Y_{m}(y) d y .
$$

This is easily evaluated to obtain

$$
R_{n m}=\left\{\begin{array}{l}
\frac{(-1)^{n} \gamma_{m} \sinh \left(\gamma_{m} a\right)}{\gamma_{m}^{2}+\frac{n^{2} \pi^{2}}{a^{2}}}, \quad h \geqslant a \\
P_{m n}+\xi_{n} Q_{m n}, \quad h<a,
\end{array}\right.
$$

where

$$
P_{m n}=\frac{\frac{m \pi}{a} \sin \left(\frac{m \pi h}{a}\right) \cosh \left(\gamma_{n} h\right)+\gamma_{n} \sinh \left(\gamma_{n} h\right) \cos \left(\frac{m \pi h}{a}\right)}{\frac{m^{2} \pi^{2}}{a^{2}}+\gamma_{n}^{2}}
$$

and

$$
Q_{m n}=\frac{\cos \left(\frac{m \pi h}{a}\right) \gamma_{n} \sinh \left(\gamma_{n} d\right)-\frac{m \pi}{a} \sin \left(\frac{m \pi h}{a}\right) \cosh \left(\gamma_{n} d\right)-(-1)^{m} \gamma_{n} \sinh \left(\gamma_{n} d\right)}{\frac{m^{2} \pi^{2}}{a^{2}}+\gamma_{n}^{2}},
$$

with $d=b-h$.

On substituting (17)-(19) into (22) and (23) and using the orthogonality relation (8) it is found, after a little rearrangement, that

$$
\begin{aligned}
B_{n}+C_{n}= & \frac{1}{2 E_{n} \nu_{n} \sin \left(\nu_{n} \ell\right)} \\
& \times\left[J Y_{n}^{\prime}(h)+i \alpha \sum_{m=0}^{\infty}\left(F_{m}-A_{m}-D_{m}\right) \eta_{m} R_{m n}\right]
\end{aligned}
$$

and

$$
\begin{aligned}
B_{n}-C_{n}= & \frac{1}{2 E_{n} \nu_{n} \cos \left(\nu_{n} \ell\right)} \\
& \times\left[-i H Y_{n}^{\prime}(h)+\alpha \sum_{m=0}^{\infty}\left(F_{m}-A_{m}+D_{m}\right) \eta_{m} R_{m n}\right] .
\end{aligned}
$$

It is straightforward to use (30) and (31) to eliminate $B_{m}$ and $C_{m}$ from (24) and (25). It is found that 


$$
\psi_{n}=-F_{n}-\frac{2 H}{\alpha \epsilon_{n}} \Theta_{n}-\frac{2 i \alpha}{\epsilon_{n} \alpha} \sum_{m=0}^{\infty}\left(F_{m}-\psi_{m}\right) \eta_{m} \Lambda_{n m}
$$

and

$$
\chi_{n}=-F_{n}+\frac{2 J}{a \epsilon_{n}} \Upsilon_{n}+\frac{2 i \alpha}{\epsilon_{n} a} \sum_{m=0}^{\infty}\left(F_{m}-\chi_{m}\right) \eta_{m} \Omega_{n m},
$$

where $\psi_{n}=\left(A_{n}-D_{n}\right), \chi_{n}=\left(A_{n}+D_{n}\right)$, and

$$
\begin{aligned}
& \Lambda_{n m}=\sum_{j=0}^{\infty} \frac{\tan \left(\nu_{j} \ell\right)}{\nu_{j} E_{j}} R_{n j} R_{m j} ; \\
& \Theta_{n}=\sum_{j=0}^{\infty} \frac{\tan \left(\nu_{j} \ell\right)}{\nu_{j} E_{j}} R_{n j} Y_{j}^{\prime}(h) .
\end{aligned}
$$

Note that $\Omega_{n m}$ and $\Upsilon_{n}$ are given by (34) and (35), respectively, with $\tan \left(\nu_{j} \ell\right)$ replaced by $\cot \left(\nu_{j} \ell\right)$.

The constants $H$ and $J$ are determined via the edge conditions (16). It is found that

$$
H=i \alpha \frac{\sum_{n=0}^{\infty}\left(\psi_{n}-F_{n}\right) \Theta_{n} \eta_{n}}{\Delta_{H}}
$$

and

$$
J=i \alpha \frac{\sum_{n=0}^{\infty}\left(\chi_{n}-F_{n}\right) \Upsilon_{n} \eta_{n}}{\Delta_{J}}
$$

where

$$
\Delta_{H}=\sum_{m=0}^{\infty} \frac{\tan \left(\nu_{m} \ell\right)\left[Y_{m}^{\prime}(h)\right]^{2}}{\nu_{m} E_{m}},
$$

and $\Delta_{J}$ is given by (38) with $\tan \left(\nu_{m} \ell\right)$ replaced by $\cot \left(\nu_{m} \ell\right)$. Equations (32) and (33) can be solved by truncation and numerical inversion of the matrix.

\section{A LOW-FREQUENCY APPROXIMATION}

In the previous section the boundary value problem was stated and reduced, using a mode-matching procedure, to a system of equations that must be truncated and solved numerically. It is useful to be able to verify the results obtained via this approach and, for this purpose, an approximate solution is now derived under the assumption $k \bar{a} \ll 1$. The usual low-frequency approximation, see (51), for an unmodified expansion chamber (i.e., without a membrane) is derived by approximating the velocity potentials $\phi_{j}, j=0,1,2 \ldots$ by just one duct mode - the fundamental plane-wave mode. For the current problem such an approximation is valid only for the inlet and outlet ducts, that is, for $\phi_{1}$ and $\phi_{3}$. To adequately describe the presence of the membrane in the expansion chamber three duct modes are required. In Sec. II it was shown that, for all frequencies, both the first coupled mode and the plane-wave mode will always propagate. An additional mode is needed, however, in order to enforce the membrane edge condition.

It is convenient to make use of the symmetry of the duct geometry and consider the symmetric and antisymmetric subproblems separately. In both cases only the left-hand side of the system need be considered, and the conditions $\phi_{2 x}^{s}(0, y)=0$ or $\phi_{2}^{a}(0, y)=0$ are applied along the line of symmetry for the symmetric and antisymmetric cases, respectively. For the symmetric subproblem the potential in the inlet duct is approximated as

$$
\phi_{1}^{s}(x) \approx e^{i x}+A_{0}^{s} e^{-i x},
$$

while

$$
\begin{aligned}
\phi_{2}^{s}(x, y) \approx & B_{0}^{s} \cos \left(\nu_{0} x\right) Y_{0}(y)+B_{1}^{s} \cos (x) \\
& +B_{2}^{s} \cos \left(\nu_{2} x\right) Y_{2}(y),
\end{aligned}
$$

where the duct modes $Y_{0}(y)$ and $Y_{2}(y)$ are given by (5). There are four unknown coefficients, that is, $A_{0}^{s}, B_{0}^{s}, B_{1}^{s}$, and $B_{2}^{s}$ and, thus, four conditions are required. An obvious choice is the membrane edge condition,

$$
\left.\frac{\partial \phi_{2}^{s}}{\partial y}\right|_{x=-\ell, y=h}=0,
$$

which relates $B_{2}^{s}$ to $B_{0}^{s}$. In addition, the velocity flux across $x=-\ell, h<y \leqslant b$ must also be zero,

$$
\left.\int_{h}^{b} \frac{\partial \phi_{2}^{s}}{\partial x}\right|_{x=-\ell} d y=0
$$

It should be noted that this does not enforce zero normal velocity at the surface; instead, it implies that the average normal velocity is zero. Continuity of average pressure is applied at $x=-\ell, 0 \leqslant y<a$,

$$
\left.\frac{1}{a} \int_{0}^{a} \phi_{2}^{s}\right|_{x=-\ell} d y=\phi_{1}^{s}(-\ell),
$$

and, finally, it is assumed that the velocity flux at the mouth of the inlet duct is equal to that across the surface $x=-\ell, 0$ $\leqslant y<h$ within the expansion chamber,

$$
\left.\int_{0}^{h} \frac{\partial \phi_{2}^{s}}{\partial x}\right|_{x=-\ell} d y=\left.a \frac{\partial \phi_{1}^{s}}{\partial x}\right|_{x=-\ell} .
$$

Although this does not accommodate the condition of zero normal velocity at the surface $x=-\ell, a \leqslant y \leqslant h$ when $h>a$, nor model the singularity in the fluid velocity at the corner, $x=-\ell, y=a$, this type of approximation is well accepted for low frequencies. ${ }^{17}$

The coefficient of interest is $A_{0}^{s}$ and, after some algebraic manipulation, it is found that

$$
A_{0}^{s} e^{2 i \ell}=-\frac{\Delta^{s}}{\left(\Delta^{s}\right)^{*}},
$$

where the asterisk indicates the complex conjugate,

$$
\begin{aligned}
\Delta^{s}= & {\left[\gamma_{2}^{2} \nu_{0} \tan \left(\nu_{0} \ell\right)-\gamma_{0}^{2} \nu_{2} \tan \left(\nu_{2} \ell\right)\right][b-i a \cot (\ell)] } \\
& -i(b-h) \tau,
\end{aligned}
$$

and

$$
\tau=\frac{\gamma_{2}^{2} \sinh \left(\gamma_{0} a\right)}{\sinh \left(\gamma_{0} h\right)}-\frac{\gamma_{0}^{2} \sinh \left(\gamma_{2} a\right)}{\sinh \left(\gamma_{2} h\right)} .
$$

For the antisymmetric problem it is found that 


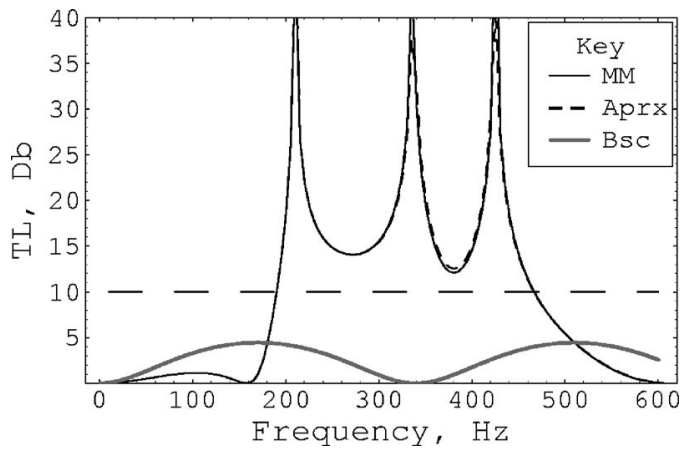

FIG. 2. Transmission loss against frequency for $\bar{a}=\bar{h}=0.05 \mathrm{~m}, \bar{b}=0.15 \mathrm{~m}$, $\bar{\ell}=0.25 \mathrm{~m}, \rho_{m}=0.1715 \mathrm{~kg} \mathrm{~m}^{-2}$, and $T=7500 \mathrm{~N} \mathrm{~m}^{-2}$.

$$
A_{0}^{a} e^{2 i \ell}=-\frac{\Delta^{a}}{\left(\Delta^{a}\right)^{*}},
$$

where

$$
\begin{aligned}
\Delta^{a}= & {\left[\gamma_{2}^{2} \nu_{0} \cot \left(\nu_{0} \ell\right)-\gamma_{0}^{2} \nu_{2} \cot \left(\nu_{2} \ell\right)\right][b+i a \tan (\ell)] } \\
& +i(b-h) \tau .
\end{aligned}
$$

The approximate solution to the full problem is obtained from the symmetric and antisymmetric subproblems simply by noting that the reflection and transmission coefficients for the fundamental modes are given by $A_{0}=\left(A_{0}^{s}+A_{0}^{a}\right) / 2$ and $D_{0}=\left(A_{0}^{s}-A_{0}^{a}\right) / 2$, respectively.

Although this is essentially a low-frequency approximation, it is found that transmission loss predictions have very good accuracy for situations in which $h=a, k \bar{a}<1$, and no more than three modes propagate in the expansion chamber. Although still good for low frequencies, the accuracy reduces as $h-a$ increases. A minor disadvantage is that the approximate solution does require numerical evaluation of the first two roots of (7).

\section{NUMERICAL RESULTS}

The usual measure of performance for an HVAC silencer is transmission loss, that is, $\mathcal{L}=-10 \log _{10}\left(P_{\text {trans }} / P_{\text {inc }}\right)$, where

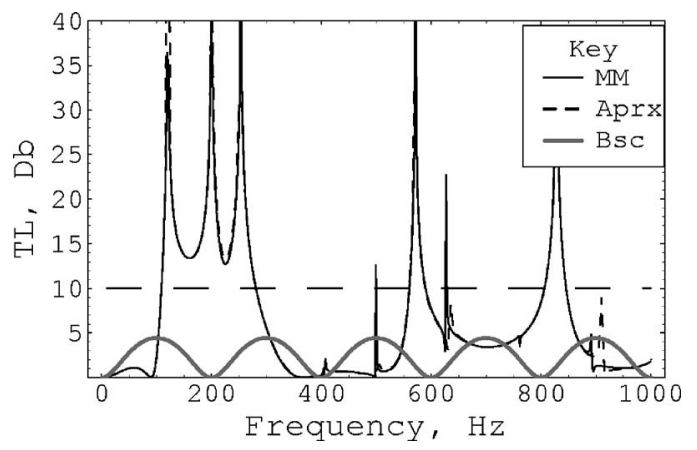

FIG. 3. Transmission loss against frequency for $\bar{a}=\bar{h}=0.085 \mathrm{~m}, \quad \bar{b}$ $=0.255 \mathrm{~m}, \bar{\ell}=0.425 \mathrm{~m}, \rho_{m}=0.20825 \mathrm{~kg} \mathrm{~m}^{-2}$, and $T=11435 \mathrm{~N} \mathrm{~m}^{-2}$.

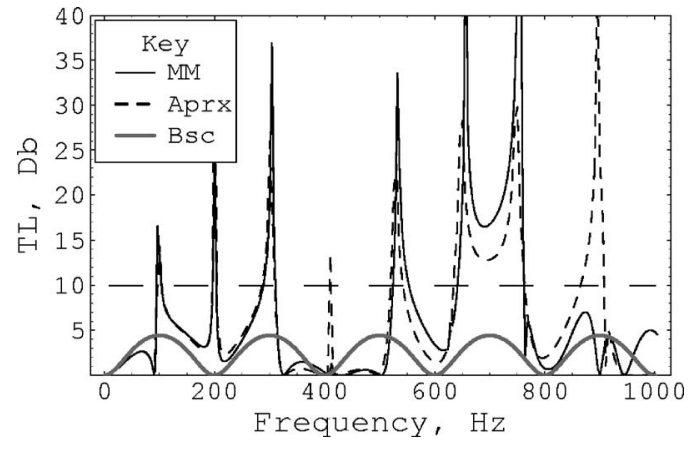

FIG. 4. Transmission loss against frequency for $\bar{a}=0.085 \mathrm{~m}, \bar{h}=0.17 \mathrm{~m}, \bar{b}$ $=0.255 \mathrm{~m}, \bar{\ell}=0.425 \mathrm{~m}, \rho_{m}=0.20825 \mathrm{~kg} \mathrm{~m}^{-2}$, and $T=11435 \mathrm{~N} \mathrm{~m}^{-2}$.

$$
P_{\text {trans }}=\Re\left\{\frac{1}{2} \sum_{n=0}^{\infty}\left|D_{n}\right|^{2} \eta_{n}^{*} \varepsilon_{n}\right\},
$$

and the incident power is $P_{\text {inc }}=1$. In this section numerical results are presented in terms of transmission loss and, where appropriate, in terms of $P_{\text {trans. }}$ In Figs. 2-15 the solid line $(M M)$ is calculated by truncating and inverting the mode-matching solution (using 20 terms). The shortdashed (Aprx) curve is obtained using the low-frequency approximation of Sec. IV. Comparison is made with the approximate transmission loss for the expansion chamber in the absence of the membrane. This solution, referred to as basic (Bsc), is plotted using the low-frequency approximate formula, ${ }^{17}$

$$
\mathcal{L}=10 \log _{10}\left\{1+\frac{1}{4}\left(\frac{\mathcal{A}_{1}}{\mathcal{A}_{2}}-\frac{\mathcal{A}_{2}}{\mathcal{A}_{1}}\right)^{2} \sin ^{2}(2 k \bar{\ell})\right\},
$$

where $\mathcal{A}_{1}$ and $\mathcal{A}_{2}$ are the cross-sectional areas of the inlet/ outlet duct and the expansion chamber, respectively. Note that, for the two-dimensional silencer considered here, $\mathcal{A}_{1}$ $=\bar{a}$ and $\mathcal{A}_{2}=\bar{b}$. For the silencer geometries considered here the basic model achieves, at best, a transmission loss in the region of $5 \mathrm{~dB}$. The horizontal line shown on the transmission loss graphs indicates $10 \mathrm{~dB}$. For the purposes of this investigation, a stopband is classified as a range of frequency for which the transmission loss exceeds $10 \mathrm{~dB}$.

It is convenient to revert to dimensional variables in order to describe the silencer parameters. The first three graphs, Figs. 2-4, deal with two silencers studied by Huang ${ }^{9}$

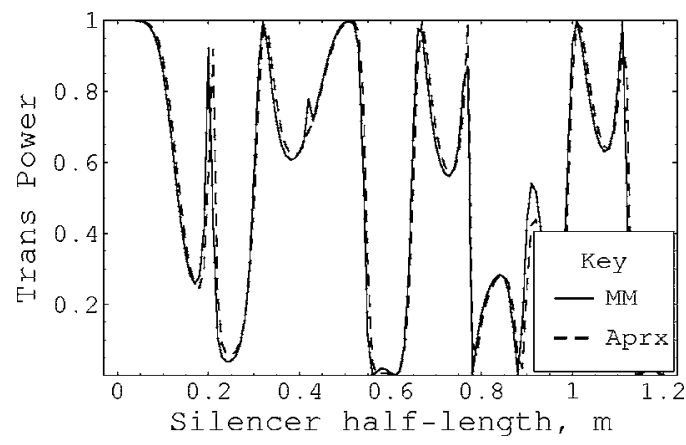

FIG. 5. Transmitted power flux against silencer half-length for $\bar{a}=\bar{h}$ $=0.15 \mathrm{~m}, \bar{b}=0.45 \mathrm{~m}, \rho_{m}=0.2 \mathrm{~kg} \mathrm{~m}^{-2}, T=3250 \mathrm{~N} \mathrm{~m}^{-2}$, and $f=200 \mathrm{~Hz}$. 


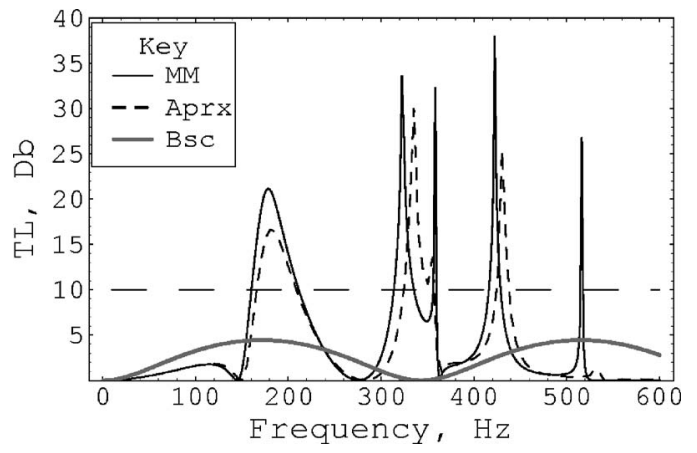

FIG. 6. Transmission loss against frequency for $\bar{a}=\bar{h}=0.15 \mathrm{~m}, \bar{b}=0.45 \mathrm{~m}$, $\bar{\ell}=0.25 \mathrm{~m}, \rho_{m}=0.2 \mathrm{~kg} \mathrm{~m}^{-2}$, and $T=3250 \mathrm{~N} \mathrm{~m}^{-2}$.

and Haung and Choy. ${ }^{18}$ The two configurations both involve small inlet ducts and comparatively long membranes with high tensions. These graphs are obtained using sound speed $340 \mathrm{~ms}^{-1}$ and fluid density $1.225 \mathrm{~kg} \mathrm{~m}^{-3}$, whereas for all remaining graphs these quantities are taken as $343.5 \mathrm{~ms}^{-1}$ and $1.2043 \mathrm{~kg} \mathrm{~m}^{-3}$, respectively.

In Fig. 2 the inlet and outlet ducts are $0.05 \mathrm{~m}$ high and the expansion chamber is of height $0.15 \mathrm{~m}$; the silencer halflength is $0.25 \mathrm{~m}$, while the membrane mass and tension are $\rho_{m}=0.1715 \mathrm{~kg} \mathrm{~m}^{-2}$ and $T=7500 \mathrm{~N} \mathrm{~m}^{-2}$, respectively. Clearly there is excellent agreement between the the mode-matching and low-frequency approximate solutions. The parameters used here are thought to be the same as that used by Huang and Choy, ${ }^{18}$ and the transmission loss curves presented in Fig. 2 agree well with the equivalent curves presented in that article.

Figure 3 shows transmission loss against frequency for a silencer with inlet/outlet ducts of height $0.085 \mathrm{~m}$ and expansion chamber of height $0.1225 \mathrm{~m}$. The membrane mass and tension are $0.20825 \mathrm{~kg} \mathrm{~m}^{-2}$ and $11435 \mathrm{~N} \mathrm{~m}^{-2}$. The parameters chosen for this silencer configuration are identical to those used by Huang ${ }^{9}$ and, again, the curves presented here are in agreement with those of that article. Although the transmission loss is comparatively low for the frequency range $550-850 \mathrm{~Hz}$, the general shape of the curve is reminiscent of a stopband and, indeed, it proves relatively easy to adjust the membrane height in order to "extract" one. This is shown in Fig. 4, where the silencer has exactly the same physical dimensions and membrane properties as for Fig. 3 other than the membrane being located within the expansion

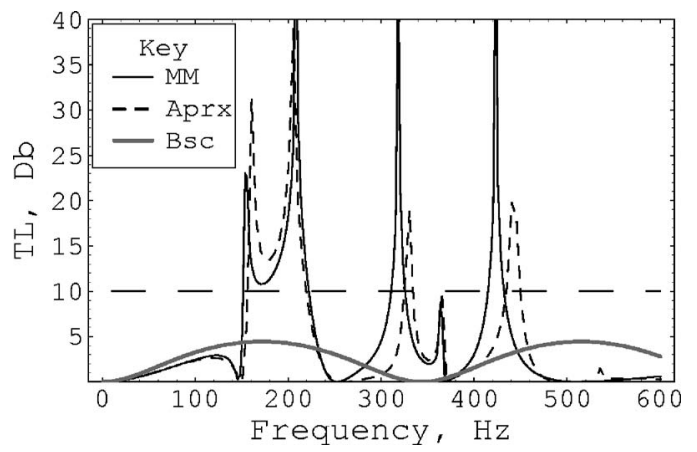

FIG. 7. Transmission loss against frequency for $\bar{a}=0.15 \mathrm{~m}, \bar{h}=0.24 \mathrm{~m}, \bar{b}$ $=0.45 \mathrm{~m}, \bar{\ell}=0.25 \mathrm{~m}, \rho_{m}=0.2 \mathrm{~kg} \mathrm{~m}^{-2}$, and $T=3250 \mathrm{~N} \mathrm{~m}^{-2}$.

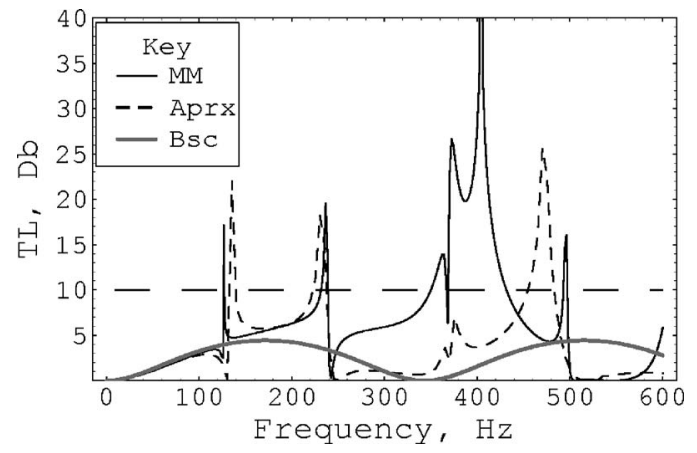

FIG. 8. Transmission loss against frequency for $\bar{a}=0.15 \mathrm{~m}, \bar{h}=0.385 \mathrm{~m}, \bar{b}$ $=0.45 \mathrm{~m}, \bar{\ell}=0.25 \mathrm{~m}, \rho_{m}=0.2 \mathrm{~kg} \mathrm{~m}^{-2}$, and $T=3250 \mathrm{~N} \mathrm{~m}^{-2}$.

chamber so that $\bar{h}=0.17 \mathrm{~m}$. The stopband which is evident in Fig. 3 for the frequency range $109-280 \mathrm{~Hz}$ has now diminished while a new stopband has emerged for $642-760 \mathrm{~Hz}$. For both Figs. 3 and 4 there is clearly a passband in the frequency range $400-500 \mathrm{~Hz}$ and the membrane height could not be adjusted to significantly improve transmission loss for this range. The mode-matching curves in Figs. 3 and 4 are validated by their close agreement with the lowfrequency approximate solution.

Both the silencer configurations considered so far have in common an aspect ratio of $\mathcal{R}=5$, where $\mathcal{R}=2 \ell /(b-a)$. Huang ${ }^{10}$ has shown that high aspect ratios tend to produce wide stopbands, but that if $\mathcal{R}$ is too large the troughs between resonant peaks in the stopband tend to drop below $10 \mathrm{~dB}$. In contrast, low aspect ratios tend to produce high but narrow stop bands. The value $\mathcal{R}=5$ is close to optimum but is impractical for the application considered here. Furthermore, it is felt that tensions as high those used for Figs. 2-4 might be difficult to sustain in practice. To be of practical use in an HVAC system the expansion chamber must ideally be low and only moderately long or, if tall, short in comparison to its height. For these reasons attention is now directed towards bigger ducts with lower aspect ratios and membranes with lower tensions. We restrict our attention to a stainlesssteel membrane of thickness $0.025 \mathrm{~mm}$ (thus $\rho_{m}=8050$ $\times 0.025 \times 10^{-3} \approx 0.2 \mathrm{~kg} \mathrm{~m}^{-2}$ ) and tension $3250 \mathrm{~N} \mathrm{~m}^{-2}$.

The issue of how to design an appropriate expansion chamber must be considered. With so many parameters involved this is not a trivial procedure. The approach taken here is to decide first on the frequency range that is to be

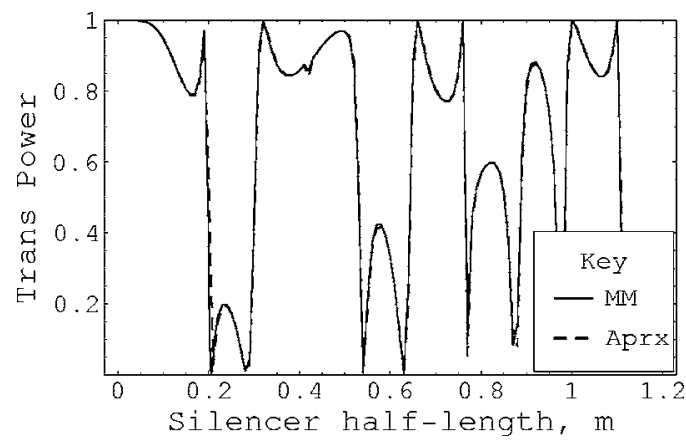

FIG. 9. Transmitted power flux against silencer half-length for $\bar{a}=\bar{h}$ $=0.15 \mathrm{~m}, \bar{b}=0.3 \mathrm{~m}, \rho_{m}=0.2 \mathrm{~kg} \mathrm{~m}^{-2}, T=3250 \mathrm{~N} \mathrm{~m}^{-2}$, and $f=200 \mathrm{~Hz}$. 


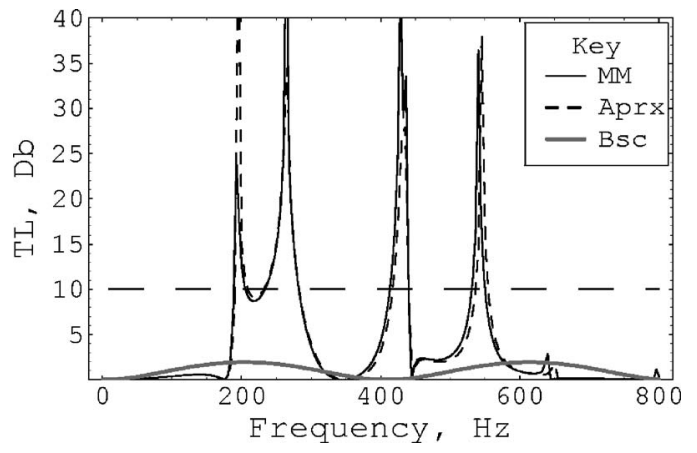

FIG. 10. Transmission loss against frequency for $\bar{a}=\bar{h}=0.15 \mathrm{~m}, \bar{b}=0.3 \mathrm{~m}$, $\bar{\ell}=0.21 \mathrm{~m}, \rho_{m}=0.2 \mathrm{~kg} \mathrm{~m}^{-2}$, and $T=3250 \mathrm{~N} \mathrm{~m}^{-2}$.

stopped, say 180-220 Hz. Then, choose the heights for the inlet/outlet ducts and expansions chamber. In Figs. 5-8 the heights for the inlet/outlet ducts and expansion chamber are 0.15 and $0.45 \mathrm{~m}$, respectively. The height of a typical HVAC duct is in the range $0.3-0.45 \mathrm{~m}$ and thus the device is an appropriate size to form a component of a hybrid silencer. It is envisaged that such a component would lie within the HVAC duct, sandwiched between panels of porous material. Length is thus an important issue- to be of practical use the device must not be long. The membrane mass and tension have already been selected so, in order to select the appropriate silencer length, the proportion of transmitted power, given by (50), is plotted with $\bar{h}=\bar{a}$ against silencer halflength for a frequency of $200 \mathrm{~Hz}$. It is clear from Fig. 5 that the transmitted power is low when $\bar{\ell} \approx 0.25 \mathrm{~m}$ and near zero when $\bar{\ell}=0.6 \mathrm{~m}$. The shorter length, which gives an aspect ratio $\mathcal{R}=1.67$, is selected for Figs. $6-8$.

Figure 6 shows transmission loss against frequency for $\bar{h}=\bar{a}$. Although the transmission loss is reasonably good in the range $160-210 \mathrm{~Hz}$, the curve does not have the same characteristic shape of the stopbands formed between two or three resonant frequencies (see Figs. 2-4). This is due to the fact that at the selected length the transmitted power flux, although small, was not close to zero (see Fig. 5). Figure 7 shows the effect altering the position of the membrane. In this case $\bar{h}=0.24 \mathrm{~m}$ and the stopband is more clearly defined and is wider, now covering the frequency range $153-220 \mathrm{~Hz}$, which is an increase of $34 \%$. The stopband can be broadened further by increasing $\bar{h}$ to $0.25 \mathrm{~m}$; however,

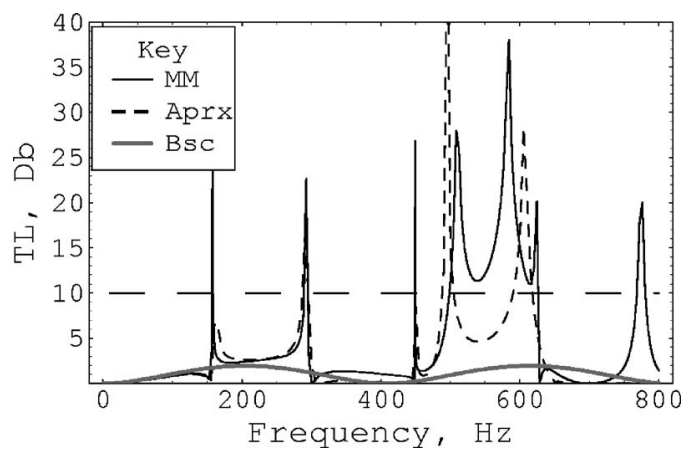

FIG. 11. Transmission loss against frequency for $\bar{a}=0.15 \mathrm{~m}, \bar{h}=0.25 \mathrm{~m}, \bar{b}$ $=0.3 \mathrm{~m}, \bar{\ell}=0.21 \mathrm{~m}, \rho_{m}=0.2 \mathrm{~kg} \mathrm{~m}^{-2}$, and $T=3250 \mathrm{~N} \mathrm{~m}^{-2}$.

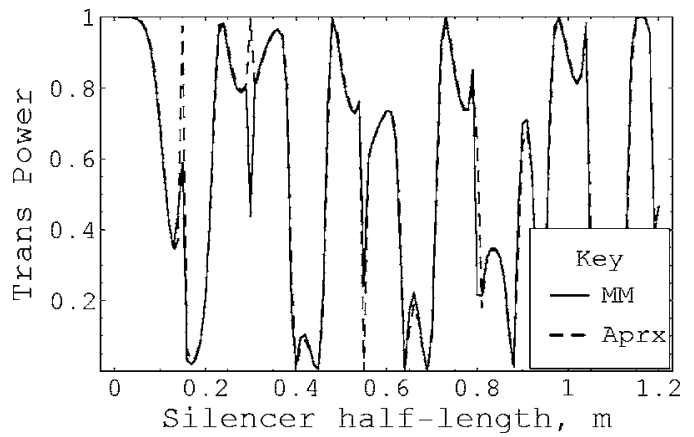

FIG. 12. Transmitted power flux against silencer half-length for $\bar{a}=\bar{h}$ $=0.15 \mathrm{~m}, \bar{b}=0.3 \mathrm{~m}, \rho_{m}=0.2 \mathrm{~kg} \mathrm{~m}^{-2}, T=3250 \mathrm{~N} \mathrm{~m}^{-2}$, and $f=300 \mathrm{~Hz}$.

this is at the cost of a small reduction in transmission loss at the center of the stopband. In both Figs. 6 and 7 the possibility of a stopband in the region $300-450 \mathrm{~Hz}$ is suggested by the shape of the curve. This stopband is extracted by moving the membrane higher into the expansion chamber so that $\bar{h}=0.385 \mathrm{~m}$. The new stopband, shown in Fig. 8, embraces the frequencies $370-434 \mathrm{~Hz}$ but, of course, the emergence of this stopband is at the expense of the first. It is clear, however, that the overall performance of the silencer with the membrane raised to this higher level is significantly better than that achieved by the basic model (i.e., the expansion chamber without membrane) for frequencies in the range 260-460 Hz.

In the next three figures the height of the inlet duct, the membrane mass, and membrane tension are as for Figs. 5-8. The height of the expansion chamber, however, is reduced to $0.3 \mathrm{~m}$. Figure 9 shows the proportion of transmitted power against silencer half-length for a frequency of $200 \mathrm{~Hz}$. The transmitted power is near zero when $\bar{\ell} \approx 0.21 \mathrm{~m}$, and this value is used for Figs. 10 and 11. The resulting silencer has an aspect ratio of $\mathcal{R}=2.8$ and transmission loss against frequency is shown, for the case $\bar{h}=\bar{a}$, in Fig. 10. Although there is a clear spike in transmission loss at $200 \mathrm{~Hz}$ (as predicted by Fig. 9), the stopband falls below $10 \mathrm{~dB}$ in the region $210-230 \mathrm{~Hz}$. The membrane height cannot be adjusted to improve this since raising the membrane causes the transmission loss in this region to drop further. It is interesting to note that the length chosen for this silencer was such that the frequency $200 \mathrm{~Hz}$ is close to a resonant value, whereas for

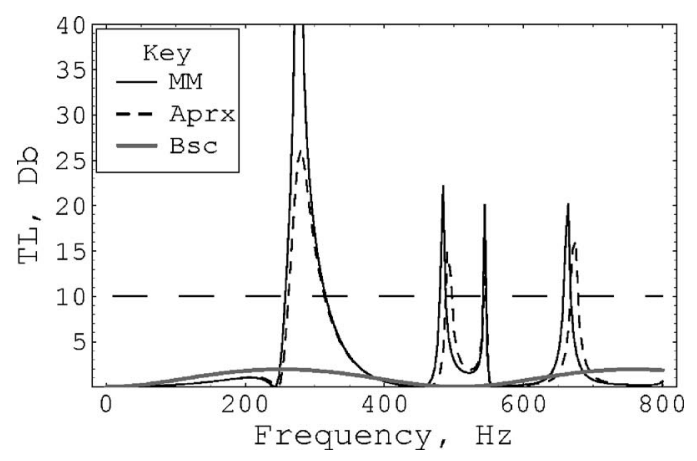

FIG. 13. Transmission loss against frequency for $\bar{a}=\bar{h}=0.15 \mathrm{~m}, \bar{b}=0.3 \mathrm{~m}$, $\bar{\ell}=0.17 \mathrm{~m}, \rho_{m}=0.2 \mathrm{~kg} \mathrm{~m}^{-2}$, and $T=3250 \mathrm{~N} \mathrm{~m}^{-2}$. 


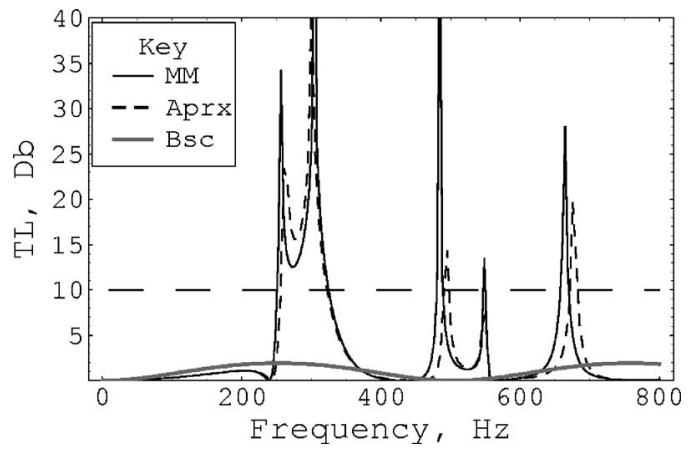

FIG. 14. Transmission loss against frequency for $\bar{a}=0.15 \mathrm{~m}, \bar{h}=0.17 \mathrm{~m}, \bar{b}$ $=0.3 \mathrm{~m}, \bar{\ell}=0.17 \mathrm{~m}, \rho_{m}=0.2 \mathrm{~kg} \mathrm{~m}^{-2}$, and $T=3250 \mathrm{~N} \mathrm{~m}^{-2}$.

Figs. 6-8 this was not the case. Figure 11 shows that, on raising the membrane further, to a height of $0.25 \mathrm{~m}$, a new stopband is formed in the frequency range $500-624 \mathrm{~Hz}$.

Figure 12 shows the proportion of transmitted power against silencer half-length for a frequency of $300 \mathrm{~Hz}$; the values of all other parameters are the same as for Fig. 9. In this case the curve shows two nonresonant minima. The value of $\ell$ is chosen to be $0.17 \mathrm{~m}$, corresponding with the lower of the two minima, and the resulting silencer has an aspect ratio of $\mathcal{R}=2.27$. Figure 13 shows the transmission loss for $\bar{h}=\bar{a}$. The shape of the stopband around $300 \mathrm{~Hz}$ is very high and narrow with a range of 260-312 Hz. Moving the membrane up a short distance into the expansion chamber, so that $\bar{h}=0.17 \mathrm{~m}$, the shopband is broadened by approximately $38 \%$. This is shown in Fig. 14. Moving the membrane further into the expansion chamber, see Fig. 15, the stopband is shifted and broadened, now encompassing all frequencies in the range $560-686 \mathrm{~Hz}$.

The final two figures are presented as a means of validating the mode-matching method for the cases $\bar{h}=\bar{a}$ and $\bar{h}$ $\neq \bar{a}$, respectively. The accuracy of a mode-matching solution is assessed by how well the matching conditions, i.e., Eqs. (20)-(23), are satisfied. Figures 16 and 17 show the absolute value of the nondimensional pressure and normal velocity at $\bar{x}=\ell$ for exactly the same silencer parameters as in Figs. 13 and 15 , respectively. The velocity $\phi_{2 x}(-\ell, y)$ is plotted for $0 \leqslant \bar{y} \leqslant \bar{b}$, while the other quantities are plotted for $0 \leqslant \bar{y}$ $\leqslant \bar{a}$. The frequency at which this comparison is made is $620 \mathrm{~Hz}$. This value was chosen because it lies in the middle

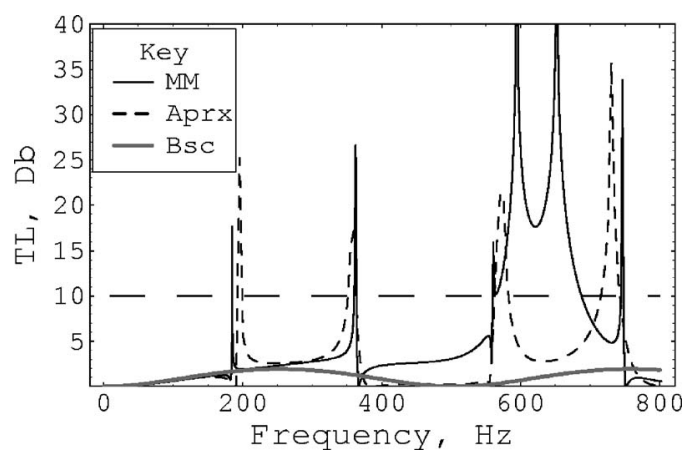

FIG. 15. Transmission loss against frequency for $\bar{a}=0.15 \mathrm{~m}, \bar{h}=0.275 \mathrm{~m}$, $\bar{b}=0.3 \mathrm{~m}, \bar{\ell}=0.17 \mathrm{~m}, \rho_{m}=0.2 \mathrm{~kg} \mathrm{~m}^{-2}$, and $T=3250 \mathrm{~N} \mathrm{~m}^{-2}$.

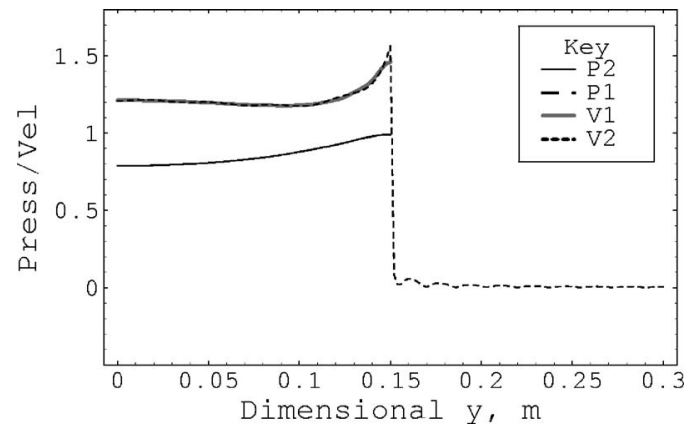

FIG. 16. Absolute value of nondimensional pressure and normal velocity against $\bar{y}, 0 \leqslant \bar{y} \leqslant \bar{a}$. Silencer parameters are $\bar{a}=\bar{h}=0.15 \mathrm{~m}, \bar{b}=0.3 \mathrm{~m}, \bar{\ell}$ $=0.17 \mathrm{~m}, \rho_{m}=0.2 \mathrm{~kg} \mathrm{~m}^{-2}$, and $T=3250 \mathrm{~N} \mathrm{~m}^{-2}$ and $f=620 \mathrm{~Hz}$.

of a range where, for Fig. 15, the low-frequency approximation and the mode-matching solution show poor agreement. In Fig. 16, where $h=a$, the pressure and normal velocity show excellent agreement [both pairs of curves overlie for $0 \leqslant y \leqslant a$ and $\phi_{2 x}(-\ell, y)=0$ for $\left.a \leqslant y \leqslant b\right]$. Thus, conditions (20)-(23) are fully satisfied. This is true at all relevant frequencies and, further, the matching conditions at $x=\ell$ are met to the same accuracy. In Fig. 17 the nondimensional pressures, that is $\phi_{1}(-\ell, y)$ and $\phi_{2}(-\ell, y)$, overlie, indicating excellent agreement. Furthermore, the normal velocity $\phi_{2 x}(-\ell, y)$ is zero for $h \leqslant y \leqslant b$ and tends rapidly to zero in the region $a \leqslant y \leqslant h$. For $0 \leqslant y \leqslant a$ the normal velocities, $\phi_{1 x}(-\ell, y)$ and $\phi_{2 x}(-\ell, y)$, are not in close agreement. Although the two curves oscillate around their mean value and the amplitude of oscillation reduces significantly as $y \rightarrow 0$, close to the corner $(y=a)$ the solutions diverge. As expected from the singular nature of the corner flow, this situation is not improved by increasing the number of terms used when truncating and inverting the mode-matching equations (20 terms were used here). Nevertheless, the normal velocities show a satisfactory level of agreement. Altering the frequency at which these comparisons are made does not significantly alter the accuracy. Furthermore, the same level of accuracy is observed at the outlet interface, that is at $x=\ell$.

To summarize, for $h \geqslant a$, the mode-matching solution satisfies (20) and (21) and, also,

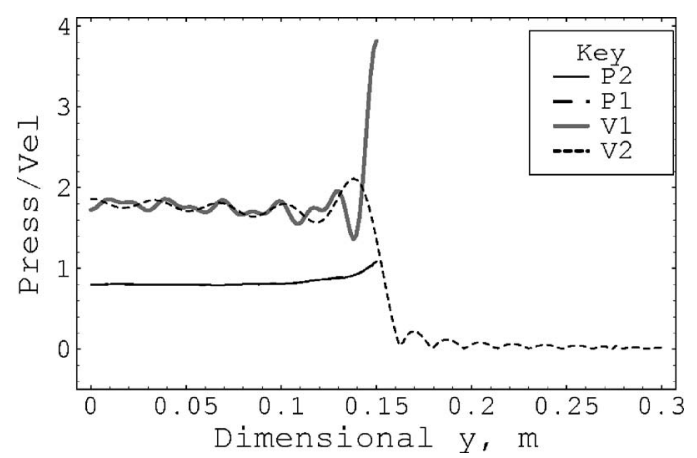

FIG. 17. Absolute value of nondimensional pressure and normal velocity against $\bar{y}, 0 \leqslant \bar{y} \leqslant \bar{a}$. Silencer parameters are $\bar{a}=0.15 \mathrm{~m}, \bar{h}=0.275 \mathrm{~m}, \bar{b}$ $=0.3 \mathrm{~m}, \bar{\ell}=0.17 \mathrm{~m}, \rho_{m}=0.2 \mathrm{~kg} \mathrm{~m}^{-2}$, and $T=3250 \mathrm{~N} \mathrm{~m}^{-2}$ and $f=620 \mathrm{~Hz}$. 


$$
\phi_{2 x}( \pm \ell, y)=0, \quad h \leqslant y \leqslant b .
$$

The normal velocities, however, are not in exact agreement for $0 \leqslant y \leqslant a$ because a Fourier cosine series cannot accurately represent an integrable singularity of the type present in the fluid velocity at $x= \pm \ell, y=a$. In view of the fact that the systems of equations derived by mode-matching automatically conserve power, ${ }^{15,14}$ it is worthwhile asking what global condition the normal velocities satisfy at $x= \pm \ell$ when $h>a$. For the parameters and frequency ranges considered herein, it has been verified numerically that the modematching solution satisfies (52) and

$$
\left.\int_{0}^{h} \frac{\partial \phi_{2}}{\partial x}\right|_{x= \pm \ell} d y=\left.\int_{0}^{a} \frac{\partial \phi_{1}}{\partial x}\right|_{x= \pm \ell} d y .
$$

(For the parameters of Fig. 15, this condition is satisfied to five decimal places for frequencies up to $800 \mathrm{~Hz}$, reducing to three decimal places at $1800 \mathrm{~Hz}$.) This, of course, is simply continuity of velocity flux and is a weaker condition than (22) and (23). That is, if (22) and (23) are satisfied then (53) must be, but the converse is not true. Nevertheless (53) is a necessary physical condition for the problem, and it is gratifying that the mode-matching solution satisfies this for $h>a-$ even though such a condition is not explicitly built into the model.

\section{DISCUSSION}

It is well established ${ }^{8,9,18}$ that a membrane attached across the mouth of an expansion chamber can dramatically reduce the transmission of low-frequency noise along a ducting system. This article has investigated the effects of altering the height of the membrane so that it lies within the expansion chamber. Attention has been restricted to silencers with low aspect ratio and subject to low-frequency incident sound. Silencers with low aspect ratio tend to produce narrow, high stopbands when the membrane lies across the mouth of the expansion chamber, ${ }^{10}$ and it has been shown that such silencers can be "tuned" by altering the membrane position. Raising the membrane a short distance into the chamber tends to broaden the stopband; increases in stopband width of up to $38 \%$ have been observed. If the membrane is raised higher into the cavity, the frequency range of the stopband is shifted, by approximately one octave, and broadened. It has, however, also been noted that passbands exist and that these cannot be eliminated by altering the membrane position.

One of the aims of this paper was to investigate this device with a view to its use as a component in a hybrid silencer ${ }^{7}$ for HVAC ducting systems. Although there is much work to be done on this subject, the initial findings are interesting. The heights of the silencers considered in this article were consistent with dimensions of a typical HVAC duct and the half-lengths were, at most, $0.25 \mathrm{~m}$. Yet stopbands of up to $72 \mathrm{~Hz}$ were achieved at low frequencies. There are, of course, potential drawbacks to the device. As the membrane is moved up into the expansion chamber a corner is formed at the junction of the inlet duct and chamber. For lowfrequency situations, such as those considered here, this does not significantly effect the results but it is potentially more problematic for high frequencies. Also, it remains to be seen whether transmission loss remains high with the inclusion of flow-particularly as the exposed corner is a potential source of turbulence once flow is introduced. Such a device, however, has been considered with a view to incorporation inside an HVAC duct between panels of absorbent material. Judicious positioning of the absorbent panels could well eliminate problems due the the corner of the expansion chamber.

The results presented herein are closely related to the work of Huang. ${ }^{8-10,18}$ The method by which the model problem is solved, however, is quite different and lends itself to a wide range of extensions to the theory. First, although the zero displacement edge condition is used here, see (16), it is equally straightforward (if less practical) to apply the condition of zero gradient. Further, the membrane can be replaced by an elastic plate, in which case any appropriate set of edge conditions (for example, clamped, pin-jointed, or free) can be enforced. In contrast, the membrane edge condition is built into Haung's method and cannot be altered. Although the latter point is not significant for a membrane, it becomes relevant should the membrane be replaced by an elastic plate, in which case the analysis is straightforward only for the case in which the plate edges are pin-jointed. ${ }^{19}$ Finally, Kang and Fuchs ${ }^{12}$ have suggested, in the context of architectural acoustics, that two membranes, one positioned at the mouth of the cavity and one within, yield a broader spectrum of noise reduction. While the application of their work is quite different from that considered here, the concept of using two membranes is intriguing. Although it is impossible to comment on any potential advantages in terms of tuning the device, problems due to the corner singularity would certainly be eliminated. The mode-matching method propounded herein is a highly appropriate method by which to study such a device, whereas the method used by Huang cannot easily deal with the internal membrane.

${ }^{1}$ A. McAlpine and M. J. Fisher, "On the prediction of 'buzz-saw' noise in acoustically lined aero-engine inlet ducts," J. Sound Vib. 265(1), 175-200 (2003).

${ }^{2} \mathrm{~S}$. Laugesen, "Active control of multi-modal propagation of tonal noise in ducts,” J. Sound Vib. 195(1), 33-56 (1996).

${ }^{3}$ J. K. Krüger, "The calculation of actively absorbing silencers in rectangular ducts," J. Sound Vib. 257(5), 887-902 (2002).

${ }^{4}$ J.-D. Wu and M. R. Bai, "Application of feedforward adaptive activenoise control for reducing blade passing noise in centrifugal fans," J. Sound Vib. 239(5), 1051-1062 (2001).

${ }^{5}$ S. J. Estève and M. E. Johnson, "Development of an adaptive Helmholtz resonator for broadband noise control," in Proceedings of IMECE2004, Anaheim, CA (2004).

${ }^{6}$ J. M. de Bedout, M. A. Franchek, R. J. Bernhard, and L. Mongeau, "Adaptive-passive noise control with self-tuning Helmholtz resonators," J. Sound Vib. 202, 109-123 (1997).

${ }^{7}$ A. Selemet, I. J. Lee, and N. T. Huff, "Acoustic attenuation of hybrid silencers," J. Sound Vib. 262, 509-527 (2003).

${ }^{8}$ L. Huang, "A theoretical study of duct noise control by flexible panels," J. Acoust. Soc. Am. 106, 1801-1809 (1999).

${ }^{9}$ L. Huang, "Modal analysis of a drumlike silencer," J. Acoust. Soc. Am. 112, 2014-2025 (2002).

${ }^{10}$ L. Huang, "Parametric study of a drumlike silencer," J. Sound Vib. 269, 467-488 (2004).

${ }^{11}$ E. H. Dowell and H. M. Voss, "The effect of a cavity on panel vibrations," AIAA J. 1, 476-477 (1963).

${ }^{12}$ J. Kang and H. V. Fuchs, "Predicting the absorption of open weave textiles and microperforated membranes backed by an air-space," J. Sound Vib. 
220(5), 905-920 (1999).

${ }^{13}$ J. B. Lawrie and I. D. Abrahams, "An orthogonality condition for a class of problems with high order boundary conditions; applications in sound/ structure interaction,” Q. J. Mech. Appl. Math. 52(2), 161-181 (1999).

${ }^{14}$ D. P. Warren, J. B. Lawrie, and I. M. Mohamed, "Acoustic scattering in waveguides that are discontinuous in geometry and material property," Wave Motion 36(2), 119-142 (2002).

${ }^{15}$ J. B. Lawrie and R. Kirby, "Mode-matching without root-finding: Application to a dissipative silencer," J. Acoust. Soc. Am. 119, 2050-2061
(2006).

${ }^{16}$ F. P. Mechel, "Theory of baffle-type silencers," Acustica 70, 93-111 (1990).

${ }^{17}$ A. P. Dowling and J. E. Ffowes Williams, Sound and Sources of Sound (Ellis Horwood, London, U.K., 1983).

${ }^{18}$ L. Huang and Y. S. Choy, "Vibroacoustics of three-dimensional drum silencer," J. Acoust. Soc. Am. 118, 2313-2320 (2005).

${ }^{19}$ L. Huang, "Broadband sound reflection by plates covering side-branch cavities in a duct," J. Acoust. Soc. Am. 119, 2628-2638 (2006). 